\title{
Comparative Performance Evaluation of On-Demand Multicast Routing Protocols for MANET
}

\author{
K. Kavitha \\ Department of Computer Science \& Engineering \\ Annamalai University, Annamalai Nagar, Tamil Nadu, India \\ E- Mail: kavithacseau@gmail.com
}

\begin{abstract}
A Mobile Ad hoc NETwork (MANET) is a collection of wireless nodes communicating with each other in the absence of any infrastructure. Each device in a MANET is free to move independently in any direction, and will therefore change its links to other devices frequently. Each must forward traffic unrelated to its own use, and therefore be a router. The primary challenge in building a MANET is equipping each device to continuously maintain the information required to properly route traffic. Such networks may operate by themselves or may be connected to the larger Internet. In this paper, we compare the performance of On-Demand Multicast Routing Protocol (ODMRP), Adaptive demand driven Multicast Routing Protocol (ADMR) With Efficient Geographic Multicast Routing Protocol (EGMP) under different mobility models such as Random Way Point Model, Manhattan Model and Random Drunken Model. Using these models, performance metrics such as Packet Delivery Ratio, End-to-End Delay and Control Overhead are evaluated. ODMRP dynamically builds the route and manages the group membership. In ADMR, Multicast routing state is dynamically established and maintained only for active groups and only in nodes located between multicast senders and receivers. ADMR detects the high mobility without the use of GPS or other positioning system. EGMP supports a zone-based scheme to efficiently handle the two-tier membership management, and takes advantage of the membership management structure to efficiently track the locations of all the group members. The simulation result shows that the throughput of ADMR is higher than that of ODMRP and EGMP at high mobility and EGMP is high at low mobility. End to end delay and control overhead of EGMP is higher than that of ODMRP and ADMR.
\end{abstract}

Keywords: MANET, ODMRP, ADMR, EGMP, Random Way Point Model, Random Drunken Model, Manhattan Model

\section{INTRODUCTION}

MANETs are a kind of wireless ad hoc networks that usually has a routable networking environment. The network is ad hoc because it does not rely on a preexisting infrastructure, such as routers in wired networks or access points in managed (infrastructure) wireless networks. Instead, each node participates in routing by forwarding data for other nodes, and so the determination of which nodes forward data is made dynamically based on the network connectivity. In addition to the classic routing, ad hoc networks can use flooding for forwarding the data [1].

MANET can be used in personal area networking such as cellular phone and laptop, emergency operations such as disaster relief, civilian environments such as meeting rooms and sports stadiums, military environments such as soldiers, tanks and planes.

An ad-hoc routing protocol is a convention, or standard, that controls how nodes decide which way to route packets between computing devices in a mobile ad hoc network [2]. In this paper, the protocols such as Efficient Geographic Multicast Routing protocol (EGMP), On-Demand Multicast Routing Protocol (ODMRP) and Adaptive Driven Multicast Routing Protocol (ADMR) are proposed. EGMP uses a virtual-zone-based structure to implement scalable and efficient two-tier membership management. A network wide zone-based bi-directional tree is constructed to achieve more efficient membership management and multicast delivery. The position information is used to guide the zone structure building, multicast tree construction, and multicast packet forwarding, which efficiently reduces the overhead for route searching and tree structure maintenance.

In wireless networking, On-Demand Multicast Routing Protocol is a protocol for routing multicast and unicast traffic throughout Ad-hoc wireless mesh networks. ODMRP creates routes on demand. This suffers from a route acquisition delay, although it helps to reduce network traffic. To reduce the problem of this delay, the source will send the first data packet along with the route discovery packet [3]. In ADMR, source-based forwarding trees are created whenever there is at least one source and one receiver in the network. ADMR monitors the traffic pattern of the multicast source application, and based on that it can detect link breaks in the tree, as well as sources that have become inactive and will not be sending any more data [4].

Mobility models represent the movement of nodes and their location, velocity and acceleration change over time. Such models are frequently used for simulation purposes when new communication techniques are investigated. In randombased mobility models, the mobile nodes move randomly and freely without any restrictions. To be more specific, the destination, speed and direction are all chosen randomly and independent of other nodes. This type of model is used in many simulation studies [5]. The Manhattan mobility model uses a grid topology. This mobility model was mainly proposed for the movement in urban area, where the streets are in an organized manner. In this mobility model, the 
mobile nodes move in horizontal or vertical direction on an urban map. The Manhattan model employs a probabilistic approach in the selection of nodes movements, since at each intersection a node chooses to keep moving in the same direction [6]. In this paper, we are analyzing EGMP,ODMRP and ADMR protocols by using different mobility models such as Random Way Point model, Manhattan model and Random drunken mobility model to measure the performance metrics such as throughput, delay and control overhead.

\section{MULTICAST ROUTING PROTOCOLS}

Multicasting is the one to many communication way. A multicast group contains a special host which is responsible for transmitting data packets to the other hosts in the same group. In MANET, multicasting can efficiently support a variety of applications that are characterized by close collaborative efforts. Multicast can reduce the communication costs, link bandwidth consumption, sender and router processing and delivery delay. In addition, it can provide a simple and robust communication mechanism when the receiver's individual addresses are unknown or changeable [7]. Multicast routing protocols for ad hoc networks have been proposed in order to save the network bandwidth and node resource because they are the protocols for powerful communication used in multi-hop applications and are more efficient than the approach of sending the same information from the source to each of the receivers individually.

Multicast protocols proposed for MANET can be broadly classified into two categories, namely tree based multicast and mesh-based multicast. A tree based multicast routing protocol establishes and maintains either a shared multicast routing tree or multiple source-based multicast routing trees to deliver data packets from sources to receivers of a group [8]. In an ad hoc wireless network, nodes may move freely within the field. For a pair of nodes to communicate route must be formed between intermediate nodes. For this type of network, it is very important to model nodes, positions and movement as transmitting range is generally small when compared to the size of the field. Multicast plays an important role in ad hoc networks. Various multicast protocols have been newly proposed to perform multicasting in Ad hoc Networks such as ODMRP, Core Assisted Mesh Protocol (CAMP), Ad Hoc Multicast Routing (AMRoute).

\section{MOBILITY MODELS}

Dynamic topology changes in wireless multi-hop network will cause lower network connectivity and/or lower network performance. To capture the nature of mobility of nodes in a mobile ad-hoc network (MANET), different mobility models have been proposed. The mobility models used in simulations can be roughly divided into two categories: independent entity models and group-based models. In the independent entity models, the movement of each node is modeled independently of any other nodes in the simulation. In the group mobility models, there is some relationship among the nodes and their movements throughout the cells or field. In order to thoroughly simulate a new protocol for an ad hoc network, it is imperative to use a mobility model that accurately represents the mobile nodes that will eventually utilize the given protocol. Only in this scenario it is possible to determine whether or not the proposed protocol will be useful when being implemented mobility model should attempt to mimic the movements of real mobile nodes [9]. Changes in speed and direction must occur in a reasonable manner. We choose models from different classes of motion, including random, path-based and group based movements.

Mobility models can be differentiated according to their spatial and temporal dependencies.

1. Spatial dependency: It is a measure of how two nodes are dependent in their motion. If two nodes are moving in the same direction then they have high spatial dependency.

2. Temporal dependency: It is a measure of how the present velocity (magnitude and direction) is related to previous velocity. Nodes having the same velocity have high temporal dependency.

Movements of mobile nodes considered in this class are completely uncorrelated. Each mobile node follows an individual independent mobility scenario. Random Waypoint, Manhattan and Random Drunken model belong to this class.

\section{A. Random Way Point (RWP) Model}

The Random Waypoint Mobility model includes pause times between changes in direction and/or speed. A mobile node begins by staying in one location for a certain period of time. Once the time expires; each node chooses a random destination in the simulation area and moves towards it with a random velocity [10]. The mobile node then travel towards the newly chosen destination at the selected speed. Upon arrival, the mobile node pauses for a specific time period before starting the process again.

Fig. 1 how an example traveling pattern of a mobile node using Random Waypoint Mobility Model starting at a randomly chosen position, the speed of the mobile node in the figure is uniformly chosen between 0 and $10 \mathrm{~m} / \mathrm{s}$. In most of the performance study that use the Random Waypoint Mobility Model, the mobile nodes are initially distributed randomly around the simulation area. When the simulation starts, each mobile node randomly selects one location in the simulation field as the destination.

The mobile nodes then travel towards this destination with constant velocity chosen uniformly and randomly from $\left[0, \mathrm{~V}_{\text {max }}\right]$, where the parameter $\mathrm{V}_{\max }$ is the maximum allowable velocity for every mobile node. The velocity and direction 
of a node are chosen independently of other nodes. Upon reaching the destination, the node stops for a duration defined by the 'pause time' parameter $T_{e}$. If $T_{e}=0$, this leads to continuous mobility. After this duration, it again chooses another random destination in the simulation field and moves towards it. The whole process is repeated again and again until the simulation ends. This mobility model shows that there is high variability in average mobile node neighbor percentage when using Random Waypoint model. A neighbor of a mobile node is a node that is within the mobile node's transmission range; the average mobile node neighbor percentage is the cumulative percentage of total mobile nodes that are given mobile nodes neighbor. There is a complex relationship between node speed and pause time in the Random Waypoint Mobility model.

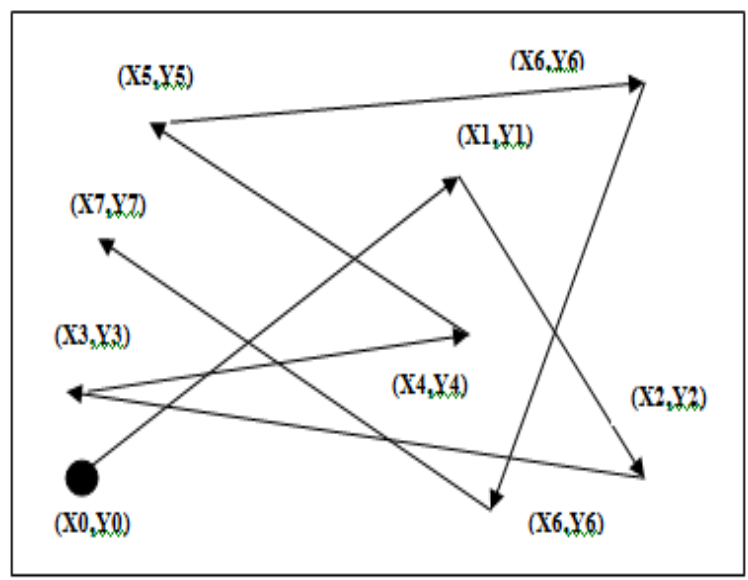

Fig. 1 Traveling pattern of mobile node using Random Waypoint Mobility Model

\section{B. Manhattan Model}

We introduce the Manhattan model to emulate the movement pattern of mobile nodes on streets defined by maps. The Manhattan map used in our study is shown in Fig. 2. The map is composed of a number of horizontal and vertical streets. Each street has two lanes for each direction. The mobile node is allowed to move along the grid of horizontal and vertical streets on the map [11]. At an intersection of a horizontal and a vertical street, the mobile node can turn left, right or go straight. This choice is probabilistic. The velocity of a mobile node at a time slot is dependent on its velocity at the previous time slot. The Manhattan mobility model is also expected to have high spatial dependence and high temporal dependence.

\section{Random Drunken Mobility Model}

In the Random Drunken mobility model, each node is assigned a random position within a field. When the node is next considered for movement, the mobility model checks all the possible directions in which the node can move to ensure that it stays within the field boundaries. The node then moves in the direction randomly chosen from the set of possible directions. Each node moves by one unit distance in that direction during the mobility period.

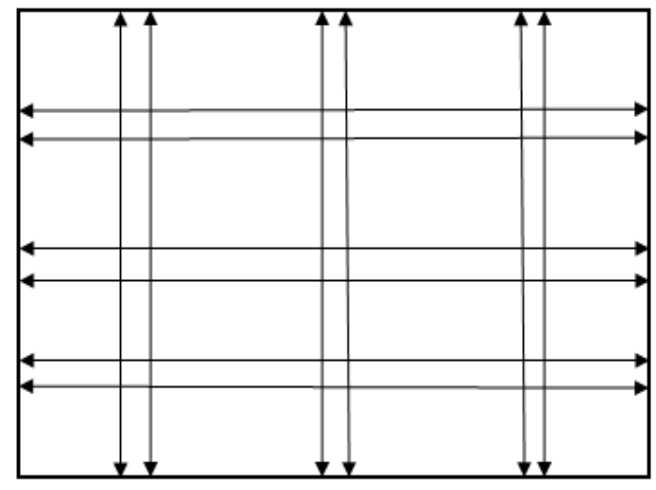

Fig. 2 Manhattan model

Here the pause time is set to zero seconds. The random drunken model periodically moves to a position chosen randomly from its immediate neighboring positions. The frequency of the change in node position is based on a parameter specified in the configuration file. This movement pattern is also known as random walk mobility model.

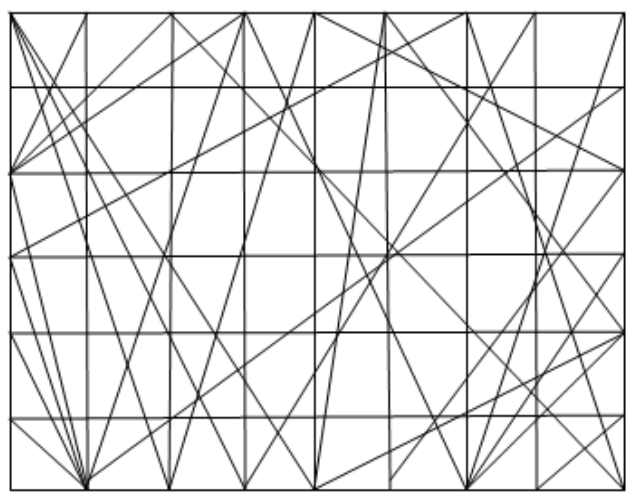

Fig. 3 Random Drunken Mobility Model

\section{ON-DEMAND MULTICAST ROUTING PROTOCOL (ODMRP)}

ODMRP is a multicast routing protocol that uses the concept of 'forwarding node' to do the multicasting. It finds some nodes to be 'forwarding node' in the whole network and only these nodes will forward multicast messages [12]. The source on-demand establishes the routes by broadcasting the Join Data message with Time to Live (TTL). This message is periodically generated to refresh both the membership and routes. Every intermediate node will add the upstream node's ID in its own routing table upon receiving this message. The message will be forwarded until it reaches a group member. The group member then creates a Join Table message and broadcasts this message to its neighbors. Every neighbor node will know itself that is on the path between the source and the group member if the next hop ID in one of the entries of the Join Table message meets its own ID. This neighbor node then establishes itself as a forwarding node. It sets the Forwarding Group Flag on. Then it builds its own Join Table message based on routing table and propagates it on 
until the message reaches the source via the shortest path as shown in Fig. 4. The mesh of forwarding nodes is established in this way. This forwarding group supports the shortest paths between any member pairs. The source can send data to all the group members with the help of the mesh. Only the forwarding nodes will forward the multicast data. It is a soft state protocol and there is no need for the group members to send explicit messages to leave the group. Members can stop working at any time and this change can be detected by the periodic refreshment. If nodes in the network have access to geographical information through equipment like GPS, ODMRP then can adapt to node movements by utilizing mobility prediction. With the mobility prediction method, the protocol becomes more resilient to topology changes.

Mobile nodes forward non-duplicated data packets if they are forwarding nodes. Since all forwarding nodes relay data, redundant paths (when they exist) are available for data packets delivery when the primary path is disconnected. ODMRP also operates as an efficient unicast routing protocol, and doesn't need support from another underlying unicast routing protocol.

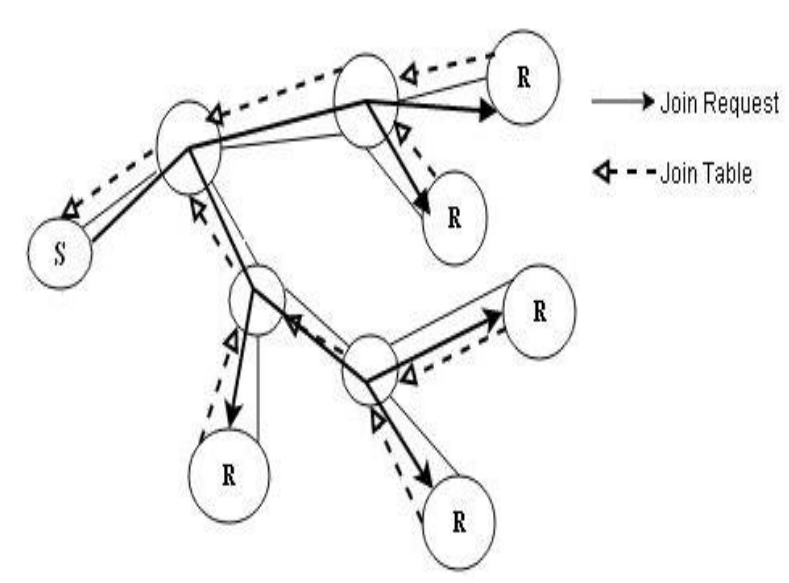

Fig. 4 Creation of mesh in ODMRP

\section{ADAPTIVE DEMAND DRIVEN MULTICAST ROUTING PROTOCOL (ADMR)}

ADMR is a multicast routing protocol that cooperates to establish and maintain forwarding state in the network to allow multicast communication. ADMR adaptively monitors the correct operation of the multicast forwarding state and incrementally repairs it when one or more receivers are forwarding nodes and it becomes disconnected from the sender. ADMR supports receivers to receive multicast packets sent by any sender, as well as the newer source specific multicast service model in which receivers may join a multicast group for only specific senders [13]. Each multicast packet is dynamically forwarded from source node $S$ along the shortest delay path through the tree to the receiver of the multicast group $G$. Each multicast packet originated by node $S$ contains a small ADMR header, including a number of fields used by the protocol in forwarding the packet and in maintaining the multicast distribution tree for node $S$ and group $G$. The sequence number in the ADMR header uniquely identifies the packet and is generated as a count of all ADMR packets flooded in any way that originated from $S$. The hop count is initialized by $S$ to 0 and is incremented by each node forwarding the packet. The ADMR header also includes the inter-packet time at which a new packet is expected from the sender $S$.

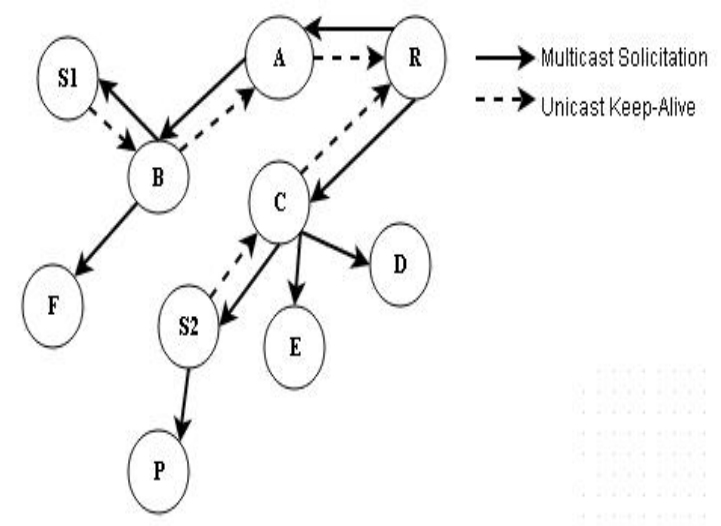

Fig. 5 Source S1 and S2 respond to receiver R's Multicast Solicitation

If the application layer at node $S$ originates no new multicast packets for $G$ within some multiple of current inter-packet time, the routing layer at $S$ begins originating "keep-alive" packets for $G$. The keep-alive is used to maintain the existing forwarding state for the multicast distribution tree for $S$ and $G$. Absence of data packets and keep-alive is an indication of forwarding tree disconnection. When any source $S$ for multicast group $G$ receives the Multicast Solicitation packet (or the single source, in the case of a source-specific multicast group join), the source replies to the Multicast Solicitation to advertise to $R$ its existence as a sender for the group. This reply may take one of two forms. If the next scheduled network flood of an existing multicast data packet is to occur soon, $S$ may choose to advance the time for this network flood and use it as the reply for the Multicast Solicitation from $R$. The other form that this reply may take is for $S$ to send an ADMR keep-alive packet unicast to $R$, following the path taken by $R$ 's Multicast Solicitation packet, as it traveled toward $S$ as shown in Fig. 5.

\section{EFFICIENT GEOGRAPHIC MULTICAST ROUTING PROTOCOL (EGMP)}

EGMP supports reliable membership management and multicast forwarding through a two-tier virtual zone-based structure. At the lower layer in reference to a predetermined virtual origin, the nodes in the network selforganize themselves into a set of zones as shown in Fig. 6; a leader is elected in a zone to manage the local group membership. At the upper layer, the leader serves as a representative for its zone to join or leave a multicast group as required [14]. The network terrain is divided into square zones as shown in Fig. 6. r: Zone size, the length of a side of the zone square. The zone size is set to $r \leq \mathrm{rt} / \sqrt{ } 2$, where rtis the transmission range of the mobile nodes. 


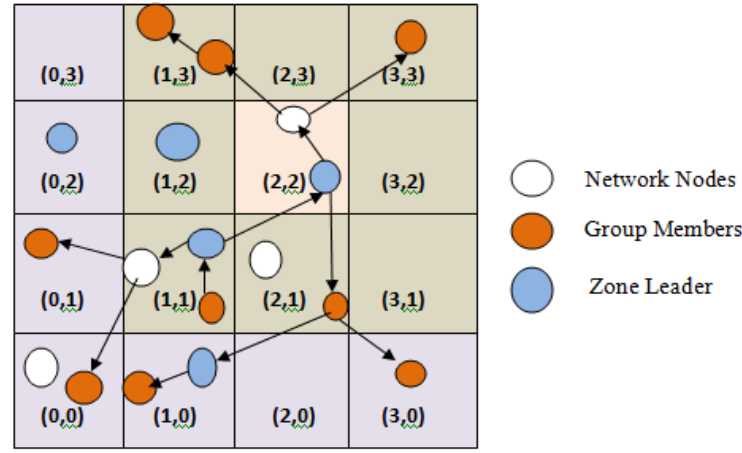

Fig. 6 Zone structure and multicast session example

To reduce intra-zone management overhead, the intra-zone nodes can communicate directly with each other without the need of any intermediate relays.

\section{PERFORMANCE METRICS}

The most important of routing performance metrics are packet delivery ratio, end to end delay and control overhead.

\section{A. Packet delivery Ratio (PDR)}

This is the ratio of total number of packets successfully received by the destination nodes to the number of packets sent by the source nodes throughout the simulation.

$P D R=\frac{\text { Numberofreceived packets }}{\text { Numberofsent packets }}$

\section{B. End to End Delay}

Delay is the time between when a message (CBR data packet) was sent and when it was received.

Average end to end delay

$$
=\sum\left(\text { Time }_{\text {received }}-\text { Time }_{\text {Sent }}\right)
$$

\section{Control Overhead}

Control overhead is the ratio of the number of control bytes transmitted per data byte delivered.

$$
\text { Control Overhead }=\frac{\text { Number of control bytes transmitted }}{\text { Data byte delivered }}
$$

\section{SIMULATION RESULTS AND PERFORMANCE COMPARISON}

We use the simulation model based on Ns2. In our Simulation the simulated traffic is Constant Bit Rate (CBR) traffic. For each simulation, we use 100 nodes, randomly placed over a square field whose length and width is 750 meters. For the Manhattan model, nodes may only be placed on one of the streets. We run each simulation for 250 seconds and we average the results of 5 simulations for each data point.

\section{PERFORMANCE BASED ON MOBILITY MODELS}

In EGMP, ODMRP and ADMR, three mobility models namely Random way point model, Manhattan model and Random drunken model are compared based on their performance metrics such as throughput, end to end delay and control overhead.

\section{A. Packet delivery Ratio}

\section{1) PDR for Random Way Point Model}

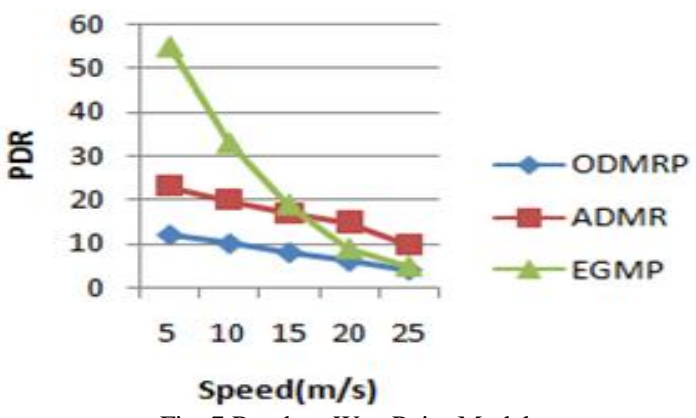

Fig. 7 Random Way Point Model

The packet delivery ratio is high in EGMP when compared to ADMR and ODMRP in Random way point model (Fig. 7), Random drunken model (Fig. 8), Manhattan Model (Fig. 9) at low mobility. PDR of ADMR is high at high mobility when compared to ODMRP and EGMP in Random waypoint and Random drunken model (Fig. 7, 8) where as in Manhattan model, ODMRP achieves higher packet delivery ratio (Fig. 9) at high mobility.

\section{2) PDR for Random Drunken Model}

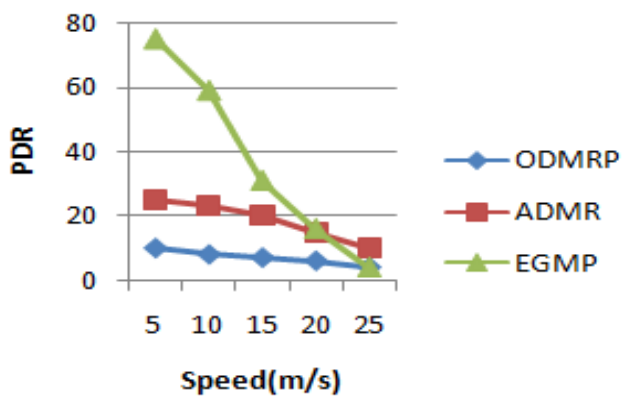

Fig. 8 Random Drunken Model

\section{3) PDR for Manhattan Model}

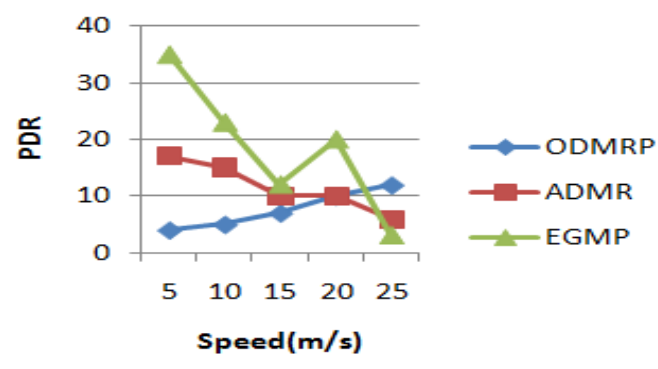

Fig. 9 Manhattan Model 


\section{B. End To End Delay}

\section{Delay for Random Way Point Model}

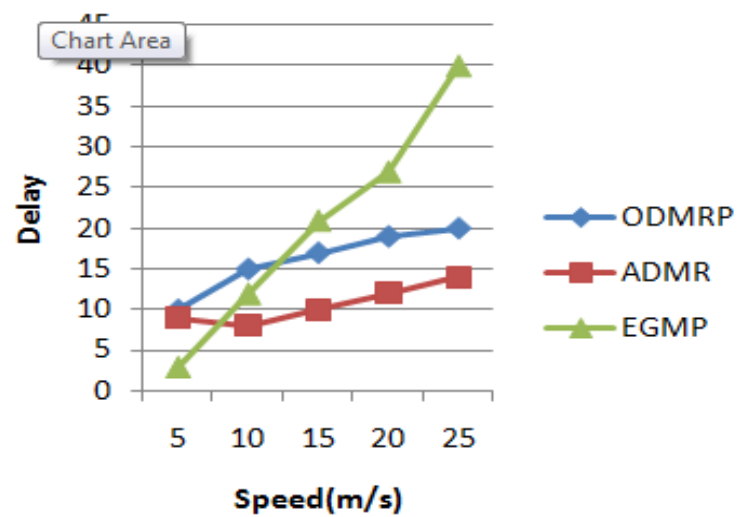

Fig. 10 Random Way Point Model

The Delay is low in EGMP When compared to ODMRP and ADMR in all the three mobility models (Fig. 10, 11, 12). At high mobility, ADMR achieves minimal delay when compared to ODMRP and EGMP in Random waypoint and Random drunken model (Fig. 10, 11). In Manhattan model, ODMRP has decreased delay when compared with ADMR and EGMP (Fig. 12).

\section{Delay for Random Drunken Model}

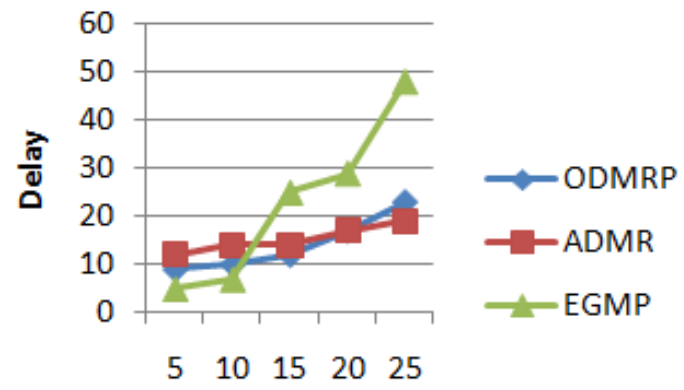

\section{$\operatorname{Speed}(\mathrm{m} / \mathrm{s})$}

Fig. 11 Random Drunken Model

3. Delay for Manhattan Model

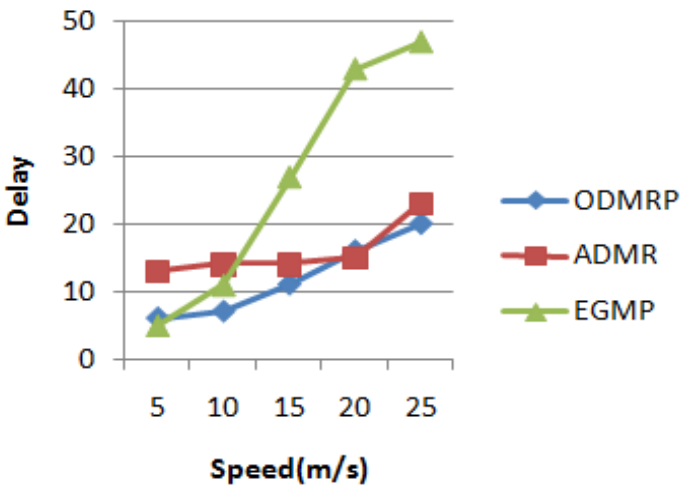

Fig. 12 Manhattan Model

\section{Control Overhead}

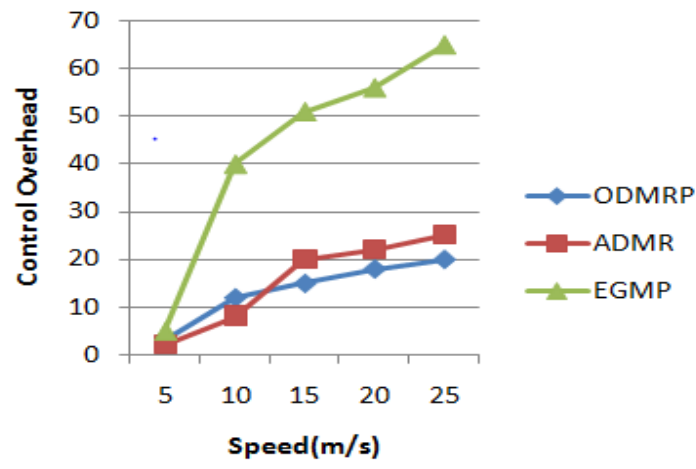

Fig. 13 Random Way Point Model

\section{Control Overhead for Random Way Point Model}

The Control messages are low in EGMP when compared to ADMR and ODMRP in all the three mobility models (Fig. $13,14,15)$ at low mobility. At high mobility, ODMRP has low control overhead when compared to both ADMR and EGMP in all the three mobility models (Fig. 13, 14, 15). Flooding has no control packets and remains relatively constant and does not increase with mobility.

\section{Control Overhead For Random Drunken Model}

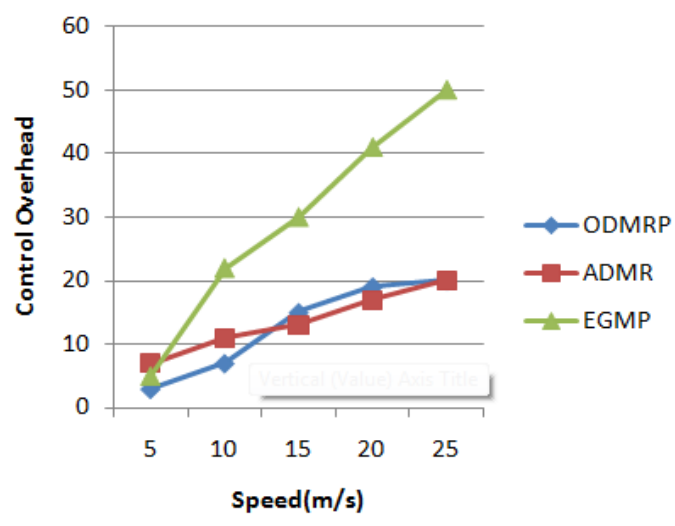

Fig. 14 Random Drunken Model

3) Control Overhead For Manhattan Model

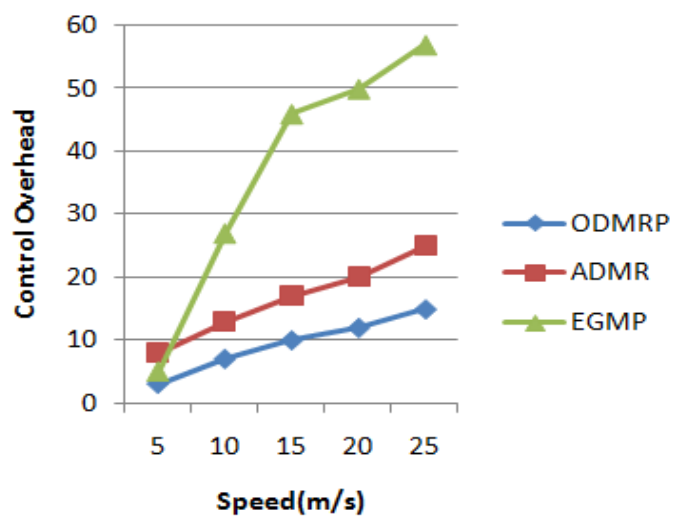

Fig. 15 Manhattan Model 


\section{CONCLUSION}

In this paper, EGMP, ODMRP and ADMR are evaluated using mobility models such as Random Way Point model, Manhattan model and Random drunken mobility model to measure the performance metrics such as Packet Delivery Ratio, End to End delay and control overhead. The simulation result shows that EGMP is able to maintain good packet delivery ratio at low mobility as compared to ADMR and ODMRP in all the three mobility models. At high mobility, packet delivery ratio is high in ADMR when compared to EGMP and ODMRP in random way point and random drunken mobility models while in Manhattan model, ODMRP achieves higher packet delivery ratio. It is observed that delay and control overhead is low for EGMP as compared to ADMR and ODMRP at low mobility under different mobility models. At high mobility, delay and control overhead is low for ADMR as compared to EGMP and ODMRP under random way point and random drunken model. In Manhattan model, ODMRP has lower delay and control overhead when compared to both EGMP and ADMR. In general, EGMP performs much better when compared with ODMRP and ADMR at low mobility.

\section{REFERENCES}

[1] Martin Appiah, "Performance Comparison of Mobility Models in Mobile Adhoc Networks", International Conference on Next Generation Computing Applications, pp. 132 - 139, 2017.

[2] Nurul I. Sarkar, and Wilford G. Lol, "A study of MANET routing protocols: Joint node density, packet length and mobility in Computers and Communications", (ISCC) 2010 IEEE Symposium, pp. 515-520, 2010.

[3] Yudhvir Singh, Yogesh Chaba, Monika Jain, and Prabha Rani, "Performance Evaluation of On-Demand Multicasting Routing Protocols in Mobile Ad hoc Networks", IEEE International Test Conference (TC), pp. 298 - 301, 2010.
[4] K.Sumathi, and A.Priyadharshini, "Energy Optimization in MANETs using On-Demand Routing Protocols", Elsevier, Vol. 45, pp. 460 $470,2015$.

[5] A.Madani, and N.Moussa, "Self-Organized Behaviour Based Mobility Models for Ad Hoc Networks", Journal of Theoretical and applied Information Technology, pp. 197 - 203, 2012.

[6] Seon Yeong Han, and Dongman Lee, "An Adaptive Hello Messaging Scheme for Neighbor Discovery in On-Demand MANET Routing Protocol", IEEE, Vol. 17, No. 5, 2013.

[7] Versha Matre, and Reena Karandikar, "Multipath routing protocol for mobile adhoc networks", Symposium on Solossal Data Analysis and Networking, pp. 1-5, 2016.

[8] R. Arun, and S. Russia, "An Efficient Multicasting Routing Protocol For MANET Using EGMP With QOS Improvements", International Journal of Advanced Research In Technology, Vol. 2, No. 2, pp. 7682, 2012.

[9] Niclolas Cooper, and Natarajan Meghanathan, "Impact of Mobility Models on Multi-path Routing in Mobile Ad hoc Networks", International Journal of Computer Networks \& Communications, Vol 2, No1, pp. 185 - 194, 2010.

[10] M.K.Pandey, and Sonika Kandari, "Random Waypoint Mobility Model based Performance estimation of MANET in terms of Average End to end delay, Jitter and Throughput for CBR Application", International Journal of Computer Applications, Vol. 106, No. 3, pp. $61-68,2014$.

[11] Satveer Kour, Hardeep singh, and Sandeep Kaur, "Performance Evaluation of Manhattan Mobility Model in Mobile Adhoc Networks", International Journal of Future Revolution in Computer Science and Communication Engineering, Vol. 4, No. 3, pp.13-18, 2018.

[12] A.Khalid, Darabkh, Ola. A.Alsukour, "Novel Protocols for Improving the Performance of ODMRP and EODMRP over Mobile Ad Hoc Networks", International Journal of Distributed Sensor Networks, Vol. 11, No. 10, pp. $102-112,2015$.

[13] Jeong -Soo kim, and Sang - Hwa Chung, "Adaptive On-demand Multi cast Routing Protocol in Mobile Ad hoc Networks", International Journal of Distributed Sensor Networks, Vol. 11, No. 11, pp. $92-101,2015$.

[14] M.Subha, and M.Manoranjani, "Modified Efficient Geographic Multicast Protocol in Multicasting over Mobile Adhoc Networks for QOS Improvements", Journal of Computer Applications, Volume 4, No. 2, pp. 57-62, 2011. 